\title{
NMR and Impedance Studies of Nanocrystalline and Amor- phous Ion Conductors: Lithium Niobate as a Model System
}

\author{
Paul Heitjans, Muayad Masoud, Armin Feldhoff and Martin Wilkening \\ University of Hannover, Institute of Physical Chemistry and Electrochemistry, Callinstr. 3-3a, \\ 30167 Hannover, Germany. Fax: +49 511762 19121; Tel: +49 511762 3187; \\ E-mail: heitjans@pci.uni-hannover.de
}

Lithium niobate has been chosen as a model system for spectroscopic studies of the influence of different structural forms and preparation routes of an ionic conductor on its ion transport properties. The Li diffusivity in nanocrystalline $\mathrm{LiNbO}_{3}$, prepared either mechanically by high-energy ball-milling or chemically by a sol-gel route, was studied by means of impedance and solid state ${ }^{7} \mathrm{Li}$ NMR spectroscopy. The Li diffusivity turned out to be strongly correlated with the different grain boundary microstructures of the two nanocrystalline samples and with the degree of disorder introduced during preparation, as seen especially by HRTEM and EXAFS. Although in both samples nanostructuring yields an enhancement of the $\mathrm{Li}$ diffusivity compared to that in coarse grained $\mathrm{LiNbO}_{3}$, the $\mathrm{Li}$ diffusivity in ball-milled $\mathrm{LiNbO}_{3}$ is much higher than in chemically prepared nanocrystalline $\mathrm{LiNbO}_{3}$. The former $\mathrm{LiNbO}_{3}$ sample has a large volume fraction of highly disordered interfacial regions which seem to be responsible for fast Li diffusion and to have a structure being very similar to that of the amorphous form. This is in contrast to the chemically prepared sample where those regions have a smaller volume fraction. 


\section{Introduction}

Solid materials with a high ionic diffusivity are of vital interest in materials science due to their potential applications as solid electrolytes, e. g., in secondary Li ion batteries..$^{1-4}$ Therefore, one of the fundamental aims in materials science is to understand the chemical and physical principles determining the transport characteristics of solids in order to design new materials with tailored diffusion properties.

A way to change the diffusion parameters of a given polycrystalline ionic conductor is to reduce its grain size down to the nanometre range, i. e., to increase the volume fraction of the interfacial regions and to introduce a large number of grain boundaries. ${ }^{5}$ As the transport properties of these regions can deviate considerably from those of the bulk material, this procedure may have a remarkable impact on the overall ionic diffusivity of the material. ${ }^{5}$ Oftentimes grain boundaries in nanocrystalline materials provide fast diffusion pathways for small cations and anions like $\mathrm{Li}^{+}$and $\mathrm{F}^{-}$, respectively, or even larger anions like $\mathrm{O}^{2-}$, so that an enhancement of the diffusivity is observed. ${ }^{6-18}$ Sometimes, however, the interfacial regions have a blocking effect resulting in a reduction of the ionic conductivity. ${ }^{19}$ This aspect is of considerable interest especially in the case of yttria-stabilized $\mathrm{ZrO}_{2}{ }^{20}$

Up to now, it is not completely clear whether the grain boundary regions of nanocrystalline ceramics prepared by different routes have similar local structures. Because of the strong relationship between the transport properties and the microscopic structure, it is of great interest to study systematically ionic diffusion in nanocrystalline materials of the same chemical composition but prepared by different routes. In the present study, lithium niobate, $\mathrm{LiNbO}_{3}$, serves as a model substance to elucidate the correlations between cation dynamics and the structural features of a nanocrystalline ceramic.

Solid-state nuclear magnetic resonance (NMR) techniques ${ }^{21,22}$ in combination with impedance spectroscopy measurements ${ }^{5,23}$ were used to probe microscopic as well as macroscopic Li diffusion parameters of $\mathrm{LiNbO}_{3}$. In its single crystalline form, lithium niobate is a poor Li conductor with a very small Li diffusion coefficient. ${ }^{6,23,24}$ The same result also holds for the microcrystalline form, i.e., a polycrystalline powder sample with an average particle size in the $\mu \mathrm{m}$ range. ${ }^{6,23}$ Interest- 
ingly, the amorphous or glassy form of $\mathrm{LiNbO}_{3}$ revealed an enhancement in Li diffusivity by several orders of magnitude. ${ }^{23,25,26}$ The activation energy of Li conductivity, reflecting long-range Li diffusion, is reduced from about $1.2 \mathrm{eV}$ in the case of single crystalline $\mathrm{LiNbO}_{3}$ to about $0.6 \mathrm{eV}$ in disordered $\mathrm{LiNbO}_{3} .{ }^{23}$ By means of ${ }^{7} \mathrm{Li}$ spin-lattice relaxation NMR, probing short-range Li motion, an activation energy of about $0.3 \mathrm{eV}$ was found for amorphous $\mathrm{LiNbO}_{3} .{ }^{27}$ This is about one third of the value obtained by ${ }^{7} \mathrm{Li}$ NMR for Li diffusion in the microcrystalline form. In nanocrystalline $\mathrm{LiNbO}_{3}$ as well, Li diffusion is drastically enhanced as compared with its coarse grained counterpart. $^{23,27,28}$ The latter result holds at least for a nanocrystalline powder prepared by ball-milling with an average particle size of about $20 \mathrm{~nm} .{ }^{23,27}$ In both samples, amorphous and nanocrystalline, the Li diffusion process can be described by practically the same set of parameters, as we have shown by an NMR relaxation and lineshape study. ${ }^{31}$ Thus, it was indirectly concluded, that the interfacial regions of the nanocrystalline material prepared by ball-milling seem to have an amorphous structure. The enhanced diffusivity in amorphous $\mathrm{LiNbO}_{3}$ was tentatively ascribed to the 'free volume' enclosed in its disordered structure leading to a distribution of energy barriers and thus to diffusion pathways with low thermal activation.

The present study is aimed at the question, whether the latter observation is a common feature of amorphous and nanocrystalline materials or at least characteristic of lithium niobate. This includes the question, whether the particle size of a nanocrystalline material is the main parameter determining the transport properties or whether structural properties of the grain boundaries play an important role, too. For this purpose we have investigated two nanocrystalline samples with the same grain size but prepared by two different techniques, viz chemically via a sol-gel method ${ }^{29}$ and mechanically using high-energy ball milling ${ }^{30}$. By the first preparation route the nanocrystallites are formed from smaller building units, similar to other techniques like inert gas condensation ${ }^{31}$, chemical vapor deposition ${ }^{32}$, or pulsed electro-deposition ${ }^{33}$. In contrast to that approach, nanocrystalline materials can also be obtained by reducing the grain size of their coarse grained counterparts, e.g., by sputtering with a radio frequency field or with heavy ions ${ }^{34}$ or just by high-energy ball-milling ${ }^{5,30}$. 
In addition to a detailed structural characterization of the samples with X-ray diffraction (XRD), high-resolution transmission electron microscopy (HRTEM), and extended X-ray absorption fine structure (EXAFS) spectroscopy, the samples were investigated by differential scanning calorimetry (DSC) and thermogravimetry (TG) as well as by Raman spectroscopy.

\section{Sample preparation and characterization}

\subsection{Preparation and XRD analysis of nanocrystalline and amorphous $\mathrm{LiNbO}_{3}$}

Phase-pure nanocrystalline $\mathrm{LiNbO}_{3}$ was prepared from the microcrystalline source material which was obtained from Alfa Aesar (99.9995\%). The coarse grained $\mathrm{LiNbO}_{3}$ consists of irregularly shaped crystallites with an average particle diameter of some tens of microns. A SPEX 8000 ball mill equipped with an $\alpha-\mathrm{Al}_{2} \mathrm{O}_{3}$ vial set and a ball of about $4 \mathrm{~g}$ made of the same material was used for ball-milling. The ball-to-powder ratio was chosen to be $1: 1$, ref. 23. Although $\mathrm{LiNbO}_{3}$ is stable in air, the whole preparation was done under an Ar atmosphere to avoid reaction of the hygroscopic nanocrystalline material with water vapour or $\mathrm{CO}_{2}$. After 16 hours of ball-milling, for instance, the average crystallite size was $23 \mathrm{~nm}$. This value was calculated from the broadening of the XRD lines using the Scherrer equation. ${ }^{35}$ The XRD profiles were measured with a Philips PW 1800 diffractometer (Bragg-Brentano geometry) using $\mathrm{Cu} \mathrm{K}$-radiation. Prior to the determination of the line broadening, the $\mathrm{K}_{\alpha 1}$ and $\mathrm{K}_{\alpha 2}$ contributions were separated from each other using the correction procedure introduced by Rachinger. ${ }^{36}$ The effect of ball milling on the XRD profiles is shown in Fig. 1(a). A Rietveld structure refinement showed that the sample milled for $16 \mathrm{~h}$ contains at most $5 \%$ of crystalline alumina due to abrasion of the vial set and the ball during the milling procedure. As compared to the influence of the different structural forms of $\mathrm{LiNbO}_{3}$ (cf. Fig. 5) this small admixture of $\mathrm{Al}_{2} \mathrm{O}_{3}$ has only a negligible effect on the ionic conductivity. For milling times equal or longer than $16 \mathrm{~h}$ the broadened XRD lines are superimposed on some broad background humps being characteristic of amorphous $\mathrm{LiNbO}_{3}$ (see below and XRD pattern at $473 \mathrm{~K}$ of Fig. 1 (b)). Thus, the XRD results indicate already that this nanocrystalline $\mathrm{LiNbO}_{3}$ sample seems to be a heterogeneous mixture of a crystalline and an amorphous phase. 

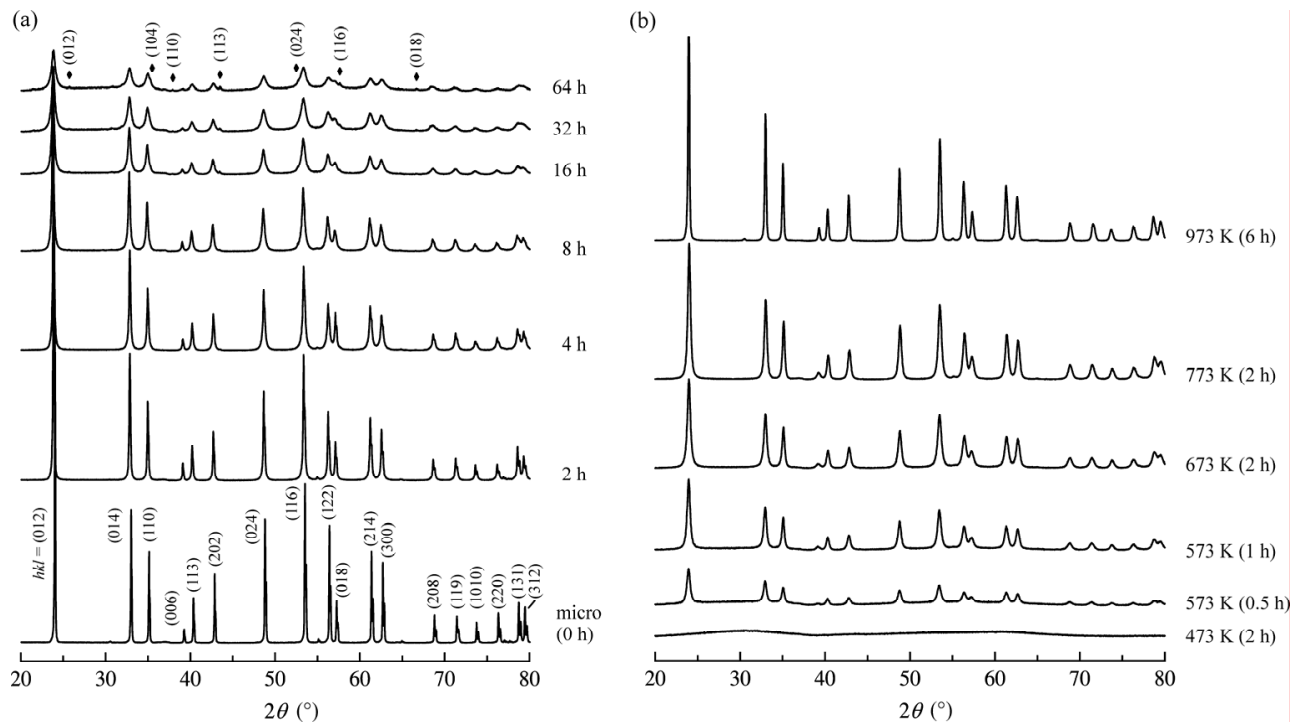

Fig. 1 (a) Effect of ball-milling for various milling times, ranging from 2 to $64 \mathrm{~h}$, on the XRD patterns of $\mathrm{LiNbO}_{3}$. Increasing the milling time results in a broadening of XRD lines due to the decrease of particle size. The $h k l$ indices of $\alpha-\mathrm{Al}_{2} \mathrm{O}_{3}$ are shown in the XRD pattern of the sample milled for $64 \mathrm{~h}$. After $16 \mathrm{~h}$ ball-milling an average particle size of $23 \mathrm{~nm}$ is reached. (b) Heat treatment of amorphous $\mathrm{LiNbO}_{3}$ prepared by complete hydrolysis of the lithium niobium double alkoxide. The XRD patterns were recorded after different periods of calcination at the indicated temperatures (in a progressive way). The XRD pattern for $773 \mathrm{~K}(1 \mathrm{~h})$ represents sol-gel prepared nanocrystalline $\mathrm{LiNbO}_{3}$ with an average particle size of about $27 \mathrm{~nm}$.

For the preparation of the other nanocrystalline sample using a sol-gel technique, amorphous $\mathrm{LiNbO}_{3}$ was synthesized by the double alkoxide route. ${ }^{29}$ Equimolar ratios of lithium ethoxide (Aldrich, $1 \mathrm{M}$ solution in abs. ethanol) and niobium ethoxide (Alfa Aesar, neat liquid, 99.999 \%) were dissolved in abs. ethanol ( $0.2 \mathrm{M}$ each). The solution was refluxed for $24 \mathrm{~h}$ at $352 \mathrm{~K}$ to prepare lithium niobium double alkoxide. Up to this point the whole preparation was done under inert gas atmosphere. After that, the double alkoxide was completely hydrolyzed with 7.5 equiv. of deionized water dissolved in abs. ethanol (2 M). The so obtained lithium niobium hydroxide hydrated gel was filtered and dried. Calcination for $2 \mathrm{~h}$ at $473 \mathrm{~K}$ under $\mathrm{O}_{2}$ flow yields $\mathrm{X}$-ray amorphous $\mathrm{LiNbO}_{3}$ (see Fig. 1 (b), XRD pattern at bottom). The pattern shows no sharp XRD lines; the broad background humps are characteristic of amorphous $\mathrm{LiNbO}_{3}$.

Another amorphous $\mathrm{LiNbO}_{3}$ sample which was used for the EXAFS investigations (see sec. 2.4) was prepared in a similar way. ${ }^{27}$ But, instead of being completely hydrolyzed, the double alkoxide solution was only partially (1/3) hydrolyzed. The resultant gel was dried for several hours in an oxygen atmosphere at $470 \mathrm{~K}$, followed by a short-time calcination for $5 \mathrm{~min}$ at $620 \mathrm{~K}$ in order to 
burn alkyl residues. The sample consists of irregularly shaped particles with diameters between 1 to $20 \mu \mathrm{m}$ and was previously used for a detailed ${ }^{7} \mathrm{Li}$ NMR relaxation study. ${ }^{27}$

Further calcination of the X-ray amorphous sample which was prepared by complete hydrolysis is shown in Fig. 1 (b). After calcination for 30 min at $573 \mathrm{~K}$ the $\mathrm{XRD}$ pattern of $\mathrm{LiNbO}_{3}$ emerges. The average particle size after heat treatment at $773 \mathrm{~K}$ is $27 \mathrm{~nm}$ when calculated from the XRD broadening using the Scherrer equation. In the following this nanocrystalline sample is called the sol-gel nanocrystalline $\mathrm{LiNbO}_{3}$. Note that the characteristic background contribution for an amorphous phase (see above) is missing in the XRD pattern for this chemically prepared sample. Thus, we conclude that the amount of amorphous regions is much less than in the mechanically prepared one. This conclusion is corroborated by a detailed analysis of TEM micrograph of these two nanocrystalline samples (see sec. 2.3). Furthermore, nanocrystalline $\mathrm{LiNbO}_{3}$ prepared by the solgel technique gives rise to relatively sharp XRD lines as compared to the milled samples.

\subsection{Sample characterization by Raman spectroscopy, DSC and TG analysis}

Raman spectra of microcrystalline and nanocrystalline $\mathrm{LiNbO}_{3}$, recorded with a Bruker optics RFS 100/S spectrometer, are shown in Fig. 2. The effect of grain size reduction can be clearly seen in Fig. 2 (a). The intensity of all Raman bands decreases with increasing milling time. Fig. 2 (b) shows the evolution of the Raman bands of $\mathrm{LiNbO}_{3}$ upon heating the hydrated double alkoxide precursor. The as-prepared gel shows very broad diffuse bands indicating a wide distribution of bond angles. For details we refer to ref. 37.

DSC curves and TG analyses were performed using a SETSYS evolution analyzer (SETARAM). The DSC curve of the as-prepared gel shows a characteristic endothermic peak around $400 \mathrm{~K}$ associated with a first TG weight loss step which can be attributed to the removal of water, alcohol and loosely bonded organic groups. At $600 \mathrm{~K}$ a strong exothermic peak due to the completion of pyrolysis is detected as confirmed by a second weight loss step. After that no weight loss and no significant DSC peaks are detected anymore. It is estimated that the amorphous $\mathrm{LiNbO}_{3}$ prepared by calcination at $473 \mathrm{~K}$ for $2 \mathrm{~h}$ will continue to consist of about $4 \%$ of organic residuals. The DSC/TG curves of the samples calcined at $773 \mathrm{~K}$ and $973 \mathrm{~K}$ did not show any DSC or weight loss peaks up to $1530 \mathrm{~K}$ which is the melting point of $\mathrm{LiNbO}_{3}$. 
(a)

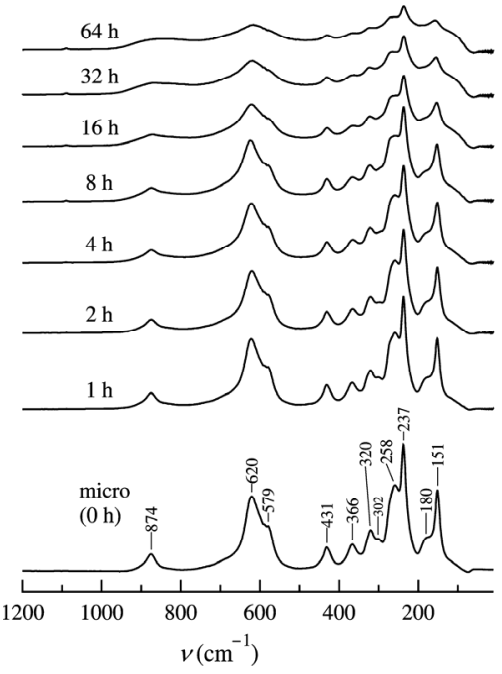

(b)

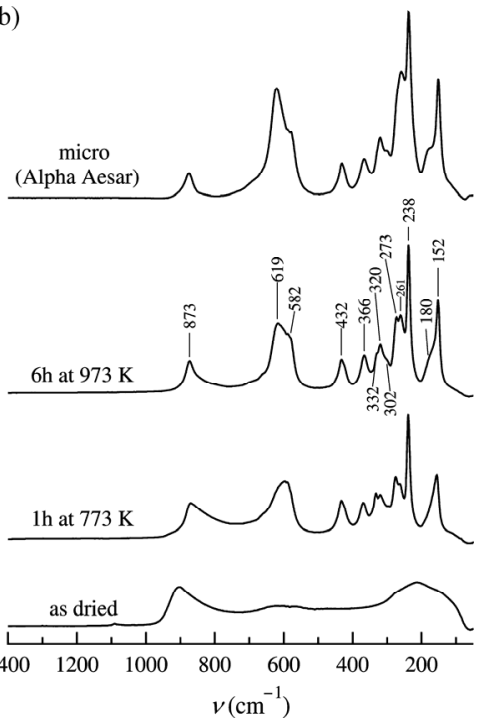

Fig. 2 (a) The effect of grain size reduction with increasing milling time on the Raman spectra of $\mathrm{LiNbO}_{3}$. (b) Raman spectra of the sol-gel precursor illustrating the evolution of nanocrystalline $\mathrm{LiNbO}_{3}$ upon heating. The Raman spectrum of the commercially available microcrystalline $\mathrm{LiNbO}_{3}$ is shown for comparison.

The micro- and single-crystalline $\mathrm{LiNbO}_{3}$ samples show no thermal changes or weight losses up to the melting point. However, the $16 \mathrm{~h}$ ball-milled $\mathrm{LiNbO}_{3}$ reveals a broad endothermic signal at temperatures extending from $350 \mathrm{~K}$ to $500 \mathrm{~K}$. This is associated with a weight-loss peak of about $3 \%$ followed by a second characteristic broad peak between $540 \mathrm{~K}$ and $700 \mathrm{~K}$ which is absent in the case of microcrystalline $\mathrm{LiNbO}_{3}$. The first one is attributed to the loss of water. Hence, contact with water and air was carefully avoided during sample preparation for impedance and NMR measurements (see above). The second peak is attributed to grain-growth of the nanocrystalline particles. Such a peak is absent for the coarse grained material and, furthermore, also absent in the case of the sol-gel nanocrystalline material. The latter observation implies that the interfacial regions differ according to the preparation route. In order to avoid any grain-growth a temperature of $450 \mathrm{~K}$ was chosen as an upper limit for all the NMR and impedance measurements.

\subsection{Characterization of nanocrystalline $\mathrm{LiNbO}_{3}$ by means of HRTEM}

HRTEM micrographs were taken at $200 \mathrm{kV}$ using a JEOL JEM-2100F-UHR field-emission microscope that provides a point resolution better than $0.19 \mathrm{~nm}$. In Fig. 2 HRTEM images of two nanocrystalline samples prepared by ball-milling for $32 \mathrm{~h}$ (Fig. 3 (a)) and chemically via the sol-gel 
method (Fig. 3 (b)) are shown. The average crystallite size of the mechanically prepared nanocrystalline sample (cf. Fig. 1 (a)) is about $20 \mathrm{~nm}$, thus, it is similar to the one milled only for $16 \mathrm{~h}$.

Fig. 3 (a) shows clearly the heterogeneous nature of ball-milled $\mathrm{LiNbO}_{3}$ consisting of a crystalline and an amorphous phase of $\mathrm{LiNbO}_{3}$. The nanocrystallites are surrounded by an amorphous grain boundary region of about $2 \mathrm{~nm}$ thickness (indicated in Fig. 3 (a) by two solid lines). Contrary to that, the chemically prepared nanocrystalline sample of $\mathrm{LiNbO}_{3}$ (Fig. 3 (b)) shows a much smaller amount of amorphous $\mathrm{LiNbO}_{3}$. The particles seem to be highly crystalline. Their grain boundary regions are somewhat disordered, however, they are much thinner compared to those of the milled sample. Some terraces and surface steps are visible.
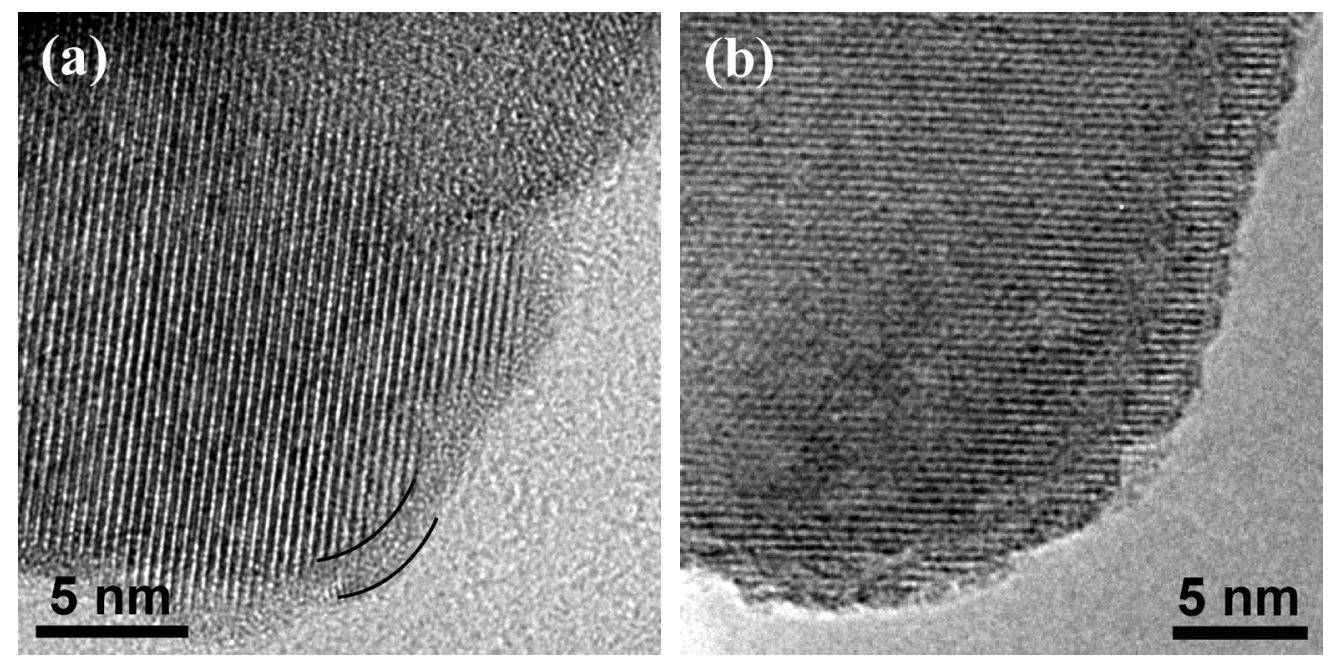

Fig. 3 (a) HRTEM micrograph of nanocrystalline $\mathrm{LiNbO}_{3}$ prepared by ball-milling for $32 \mathrm{~h}$, with an average particle size of about $20 \mathrm{~nm}$. A large amount of amorphous $\mathrm{LiNbO}_{3}$ can be seen. (b) HRTEM micrograph of nanocrystalline $\mathrm{LiNbO}_{3}$ prepared chemically via complete hydrolysis of the lithium niobium double alkoxide. Although the grain boundary regions are somewhat disordered, not much amorphous material can be detected.

\subsection{EXAFS measurements}

In addition to TEM measurements, a series of EXAFS experiments were carried out using station 9.2 at the Daresbury synchrotron radiation source in order to explore the nature of the grain boundaries of a mechanically prepared sample via high-energy ball-milling for $16 \mathrm{~h}$ which we used previously for an NMR study. ${ }^{6}$ All the measurements were done at the Nb K-edge and experimental 
data were collected in transmission mode. The minimum beam current was $150 \mathrm{~mA}$ at $2 \mathrm{GeV}$ and the beam size was $10 \mathrm{~mm}$ by $0.6 \mathrm{~mm}$. The energy selection was accomplished by a double crystal $\mathrm{Si}(220)$ monochromator. Standard ion chambers were used as detectors. The EXAFS data were analyzed using the Daresbury suite of EXAFS programs. For further details see refs. 37, 38.

(a)

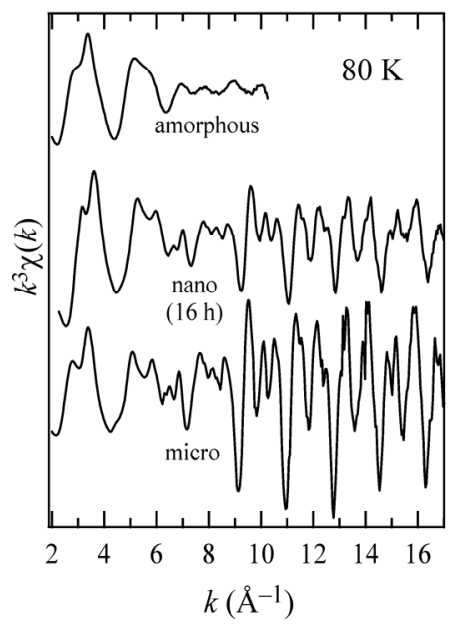

(b)

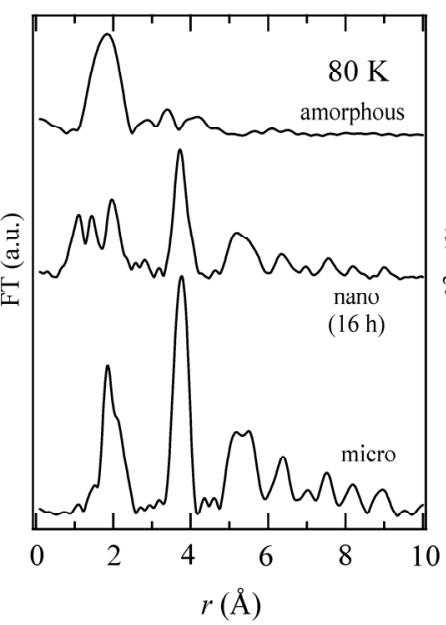

(c)

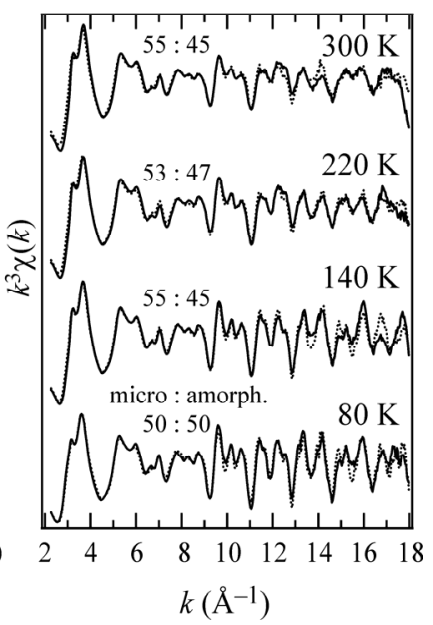

Fig. 4 (a) $\mathrm{Nb}$ K-edge EXAFS spectra for microcrystalline, mechanically prepared nanocrystalline and amorphous $\mathrm{LiNbO}_{3}$ at $T=80 \mathrm{~K}$. (b) Fourier transforms of the EXAFS spectra of the three forms of $\mathrm{LiNbO}_{3}$. (c) EXAFS spectra of the nanocrystalline sample. Data were recorded at different temperatures. Dotted curves represent the fits using a linear combination of the EXAFS spectra of microcrystalline and amorphous $\mathrm{LiNbO}_{3}$ at the same temperature. Nanocrystalline $\mathrm{LiNbO}_{3}$ can be described by a linear combination with a ratio of approximately $1: 1$ of microcrystalline and amorphous $\mathrm{LiNbO}_{3}$.

EXAFS data of amorphous $\mathrm{LiNbO}_{3}$ being prepared via partial hydrolysis and results of microcrystalline $\mathrm{LiNbO}_{3}$ (Aldrich, $99.999 \%$, see ref. 6) were used as internal references for analyzing the experimental EXAFS spectra of the ball-milled $\mathrm{LiNbO}_{3}$ at various temperatures. The data obtained at $80 \mathrm{~K}$ are presented in Fig. 4 (a) and their Fourier transforms are shown in Fig. 4 (b). A linear combination of EXAFS spectra of microcrystalline and amorphous $\mathrm{LiNbO}_{3}$ is fitted to the spectra of the nanocrystalline sample ( $c f$. Fig. 4 (a) and (c)). The fit shows that the nanocrystalline material consists in about equal shares of crystalline and amorphous $\mathrm{LiNbO}_{3}$. Thus, the findings obtained by XRD and TEM measurements are corroborated by the EXAFS data. Obviously, the ballmilled samples of $\mathrm{LiNbO}_{3}$ can be regarded as structurally heterogeneous materials. The nanocrys- 
tallites seem to be embedded in an amorphous matrix of $\mathrm{LiNbO}_{3}$. Similar results have been obtained recently by Chadwick and coworkers from an EXAFS measurement at $300 \mathrm{~K}^{39}$

\section{Experimental}

\subsection{Impedance spectroscopy}

Most of the impedance measurements were carried out in the frequency range from $v=5 \mathrm{~Hz}$ to $13 \mathrm{MHz}$ using an RF impedance analyzer HP 4192A. The impedance detection range was $0.1 \mathrm{~m} \Omega-$ $1 \mathrm{M} \Omega$. A home-built impedance cell was used for the measurements. It was placed in a horizontaltube furnace controlled by an Eurotherm 818 programmable temperature unit. The temperature was measured $3 \mathrm{~mm}$ away from the sample, its accuracy is better than $0.5 \mathrm{~K}$.

Due to the low conductivity of single crystalline $\mathrm{LiNbO}_{3}$ an Alpha Novocontrol high-resolution dielectric analyzer was utilised which works in the frequency range from $\nu=3 \mu \mathrm{Hz}$ to $10 \mathrm{MHz}$ and in a large impedance range, i. e., from $10^{-2}$ to $10^{14} \Omega$. A connection head (BDS 1200) served as the standard sample cell. The temperature was monitored with an accuracy of $0.3 \mathrm{~K}$ by means of a PT100 temperature unit. It is either adjusted with freshly evaporated nitrogen or with an electrical heater controlled by WinDETA (Novocontrol).

Pellets of the powder samples were prepared by applying an uniaxial pressure of about $0.75 \mathrm{GPa}$. The thickness ranged between 0.2 and $0.4 \mathrm{~mm}$. In the case of single-crystalline $\mathrm{LiNbO}_{3}$, plates with a thickness of $0.45 \mathrm{~mm}$ and an area of $15 \times 12 \mathrm{~mm}^{2}$ were used for the measurements. The surfaces of the plates were polished and sputtered with gold.

All the experiments were done either in inert gas or in dry oxygen atmosphere. The results proved to be highly reproducible, no hysteresis behaviour of the conductivity was observed when excluding any influence of water vapour on the experiments. Complex plane impedance plots were analysed by electrical equivalent circuit software.

\subsection{NMR measurements}

${ }^{7} \mathrm{Li}$ spin-lattice relaxation NMR rates $1 / T_{1}$ as a function of inverse temperature were recorded using a modified MSL 100 Bruker spectrometer at a Larmor frequency $\omega_{0} / 2 \pi$ of $77.72 \mathrm{MHz}$. The MSL 100 is connected to a tunable Oxford cryomagnet and equipped with a Kalmus $400 \mathrm{~W}$ power 
amplifier. The $90^{\circ}$ pulse length using a commercial Bruker broadband probe was about $5 \mu$ s. Relaxation rates in the laboratory frame were measured with the standard saturation recovery pulse sequence ${ }^{40}, n \times 90^{\circ}-$ delay time $t_{i}-90^{\circ}$ - acq., where a train of $(n=10) 90^{\circ}$ pulses destroys any longitudinal magnetization $M_{z}$ so that the subsequent recovery of $M_{z}$ can be detected via the last $90^{\circ}$ pulse as a function of thirty different delay times $t_{i}$ ranging between $t_{1} \rightarrow 0$ and $t_{30}>5 T_{1}$. Up to 32 scans were accumulated for each value of $t_{i}$. Magnetization transients $M_{z}\left(t_{i}\right)$ were obtained by integration of the free induction decays.

${ }^{7} \mathrm{Li}$ NMR spectra were recorded using the solid-echo pulse sequence, $\phi-t_{e}-90^{\circ}-t_{e}-$ echo, modified for a spin-3/2 nucleus, i. e., $\phi=64^{\circ}$. The time-domain data were converted into the frequency-domain by Fourier transformation starting from the echo top at $t=t_{\mathrm{e}}$. The interpulse delay $t_{\mathrm{e}}$ ranged between 10 and $100 \mu \mathrm{s}$. It was chosen such that $t_{\mathrm{e}}$ was smaller than the transverse relaxation time $T_{2}$.

\section{Results and Discussion}

\subsection{Impedance spectroscopy}

The conductivity spectra $\sigma^{\prime}(v)$ of all the different forms of $\mathrm{LiNbO}_{3}$ investigated, viz single-crystalline, microcrystalline, nanocrystalline as well as amorphous $\mathrm{LiNbO}_{3}$, consist of well-defined $\sigma_{\mathrm{dc}}{ }^{-}$ plateaus at low frequencies and a typical dispersive regime in the high-frequency range due to correlated Li jumps on shorter time scales. The spectra can be described empirically by the power law

$$
\sigma^{\prime}(v)=\sigma_{\mathrm{dc}}+A(v 2 \pi)^{S}
$$

with the exponent $s$ ranging between 0.55 and 0.77 , ref. 23. The temperature dependence of $\sigma_{\mathrm{dc}}$ is shown in Fig. 5. Each sample shows single Arrhenius behaviour

$$
T \cdot \sigma_{\mathrm{dc}}=A^{\prime} \exp \left(-E_{\mathrm{A}, \sigma} / k_{\mathrm{B}} T\right)
$$

over the respective measured temperature ranges so that we assume that no change in the conduction mechanism occurs. The lowest Li conductivity is found for single-crystalline and microcrystalline $\mathrm{LiNbO}_{3}$. It is increased by seven orders of magnitude for amorphous $\mathrm{LiNbO}_{3}$. A drastic enhancement of the Li conductivity, though somewhat less than for amorphous $\mathrm{LiNbO}_{3}$, is also found 


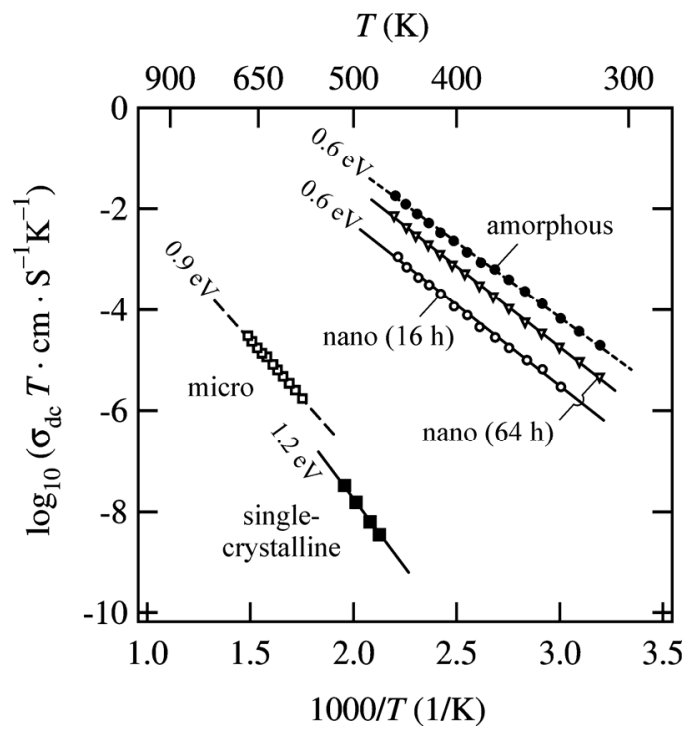

Fig. 5 Temperature dependence of $\sigma_{\mathrm{dc}}$ for amorphous $(\bullet)$ and ball-milled nanocrystalline $\mathrm{LiNbO}_{3}$ (milling time: $16 \mathrm{~h}(\circ), 64 \mathrm{~h}(\nabla)$ ). The results are compared to those obtained for commercially available microcrystalline ( $\square$, Alfa Aesar) and single-crystalline (a) $\mathrm{LiNbO}_{3}$. The microcrystalline $\mathrm{LiNbO}_{3}$ was used as source material to prepare the nanocrystalline samples. Dashed and solid lines show fits according to the Arrhenius relation of eqn. (2).

for the nanocrystalline material prepared via $16 \mathrm{~h}$ high-energy ball-milling. Whereas the diffusion process in single-crystalline and microcrystalline $\mathrm{LiNbO}_{3}$ is determined by an activation energy $E_{\mathrm{A}, \sigma}$ of about $1 \mathrm{eV}$, the corresponding values of $E_{\mathrm{A}, \sigma}$ for the nanocrystalline $(16 \mathrm{~h}$ ball-milled, $23 \mathrm{~nm}$ particle size) and amorphous forms are reduced by a factor of two resulting in activation energies of about $0.59 \mathrm{eV} . E_{\mathrm{A}, \sigma}$ refers to the long-range transport process of the charge carriers. Interestingly, both samples follow an Arrhenius relation (eqn. (2)) with the same activation energy, thus reflecting similar conduction pathways in amorphous and mechanically prepared nanocrystalline $\mathrm{LiNbO}_{3}$. With increasing milling time the data points of the nanocrystalline material are shifted towards the Arrhenius line of the amorphous sample, so that the difference between the $T \cdot \sigma_{\mathrm{dc}}(1 / T)$ values of the two materials becomes smaller than one order of magnitude.

Contrary to these observations, the behaviour of the sol-gel prepared nanocrystalline sample with an average particle size of about $27 \mathrm{~nm}$ is completely different. Fig. 6 (a) compares the $\sigma_{\mathrm{dc}}$ temperature dependence of the sol-gel nanocrystalline $\mathrm{LiNbO}_{3}$ with that of the amorphous sample prepared from the double alkoxide calcined for $2 \mathrm{~h}$ at $473 \mathrm{~K}$ and with that of a sample heat treated at 
(a)

$T(\mathrm{~K})$

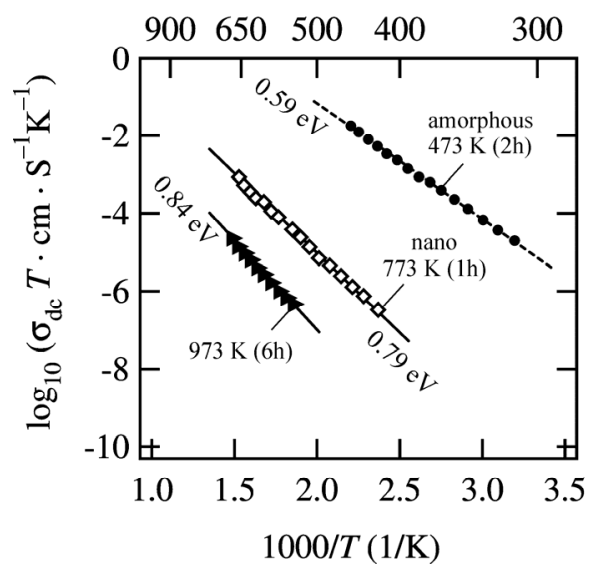

(b) $T(\mathrm{~K})$

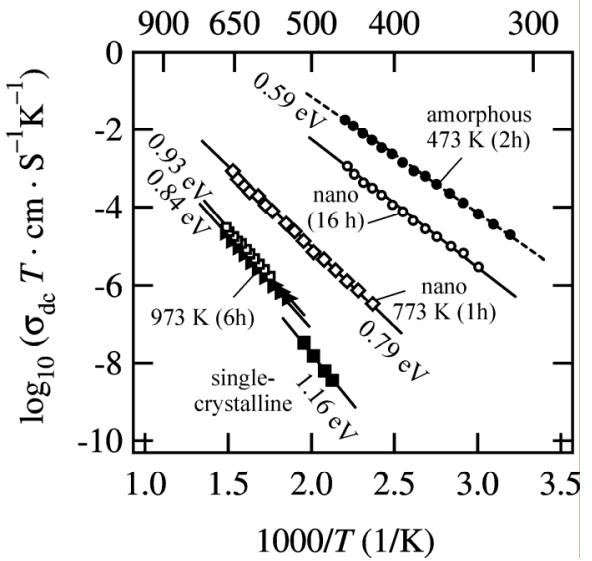

Fig. 6 Temperature dependence of the dc-conductivity of amorphous $\mathrm{LiNbO}_{3}$ in comparison with those of sol-gel prepared nanocrystalline $\mathrm{LiNbO}_{3}(773 \mathrm{~K}, 1 \mathrm{~h}, c f$. Fig. 1) and with a sample heat-treated at much higher temperature (973 K, $c f$. Fig. 1 for the corresponding XRD pattern).

$973 \mathrm{~K}$. The latter one has an average particle size of about $100 \mathrm{~nm}$. Its conductivity behaviour is nearly the same as that obtained for microcrystalline $\mathrm{LiNbO}_{3}$ which was used as the source material for high-energy ball-milling (see sec. 2.1). The data points coincide and the activation energies are very similar, viz $0.84 \mathrm{eV}$ and $0.93 \mathrm{eV}$, respectively (cf. Fig. 6 (b)). Although the conductivity for the sol-gel prepared nanocrystalline $\mathrm{LiNbO}_{3}$ is higher by two orders of magnitude when compared with the results of the sample prepared at $973 \mathrm{~K}$ and of microcrystalline $\mathrm{LiNbO}_{3}$, it is much smaller than that of the sample prepared by milling and that of amorphous $\mathrm{LiNbO}_{3}$, respectively. The chemically prepared nanocrystalline sample takes a medial position between the single-crystalline and microcrystalline form on the one hand and the ball-milled nanocrystalline and amorphous material on the other hand. The activation energy of about $0.79 \mathrm{eV}$ comes closer to the values $E_{\mathrm{A}, \sigma}$ obtained for the coarser grained materials. Starting with the amorphous sample, $E_{\mathrm{A}, \sigma}$ first increases from $0.59 \mathrm{eV}$ by about $0.2 \mathrm{eV}$ to $0.79 \mathrm{eV}$ for the chemically prepared nanocrystalline material $(27 \mathrm{~nm})$ and then to $0.84 \mathrm{eV}$ for the sample heat-treated at $973 \mathrm{~K}$ with a particle size around $100 \mathrm{~nm}$. The latter change in activation energy can be attributed to the onset of grain-growth leading to a reduction of interfacial regions in the material.

The difference in transport properties of the two nanocrystalline samples cannot be explained by different particle sizes as the average diameter of the nanometer sized grains are very similar, 
namely $23 \mathrm{~nm}$ and $27 \mathrm{~nm}$. The conductivity results imply that the nature of the interfacial regions of the chemically prepared sample are indisputably different from those present in the mechanically prepared one. Provided the microstructure of the grain boundaries is the relevant difference between both materials, it is obvious from the very similar conductivity behaviour of ball-milled nanocrystalline and amorphous $\mathrm{LiNbO}_{3}$ that the interfacial regions of the mechanically prepared material have an amorphous-like structure.

This finding is in agreement with the XRD, TEM and EXAFS results uncovering the amorphous structure of the interfacial regions. The same result was also concluded indirectly from measurements probing local Li diffusion via ${ }^{7} \mathrm{Li}$ spin-lattice relaxation NMR, previously performed in our laboratory. ${ }^{27}$ The amorphous grain boundaries, which dominate the Li transport in the ball-milled sample, represent fast conduction pathways resulting in a high Li conductivity with a low activation energy. However, these fast diffusion pathways seem to be absent in chemically prepared nanocrystalline $\mathrm{LiNbO}_{3}$. The XRD results indicate and the TEM images show rather clearly, that the chemically prepared sample is composed of nanometer sized grains with thin grain-boundary regions. Large amounts of an amorphous phase and thus fast diffusion pathways, as visible in the case of ball-milled $\mathrm{LiNbO}_{3}$, are missing. Thus, it can be easily understood why the chemically prepared nanocrystalline sample shows a relatively low conductivity being comparable to that of the microcrystalline material. In sol-gel prepared nanocrystalline samples there is no way to enhance the ionic conductivity in the same way as is easily possible for the ball-milled samples.

It should be noted, that sol-gel synthesis proves to be a highly suitable technique for the synthesis of nanosized crystallites with relatively thin and ordered interfacial regions. Quite recently, Niederberger et al. ${ }^{41}$ reported similar results for $\mathrm{LiNbO}_{3}$ prepared by a soft-chemistry route.

\subsection{Solid-echo and relaxation NMR measurements}

\subsection{1 ${ }^{7} \mathrm{Li}$ NMR lineshape analysis}

Information about structural aspects and Li dynamics can be obtained by recording ${ }^{7} \mathrm{Li}$ NMR spectra of the different forms of $\mathrm{LiNbO}_{3}$. In Fig. 7 static ${ }^{7} \mathrm{Li}$ NMR spectra at selected temperatures between $153 \mathrm{~K}$ and $673 \mathrm{~K}$ are shown for the amorphous, the two nanocrystalline and the microcrystalline samples. As ${ }^{7} \mathrm{Li}$ is a quadrupole nucleus with a spin quantum number of $3 / 2$, it is expected that 

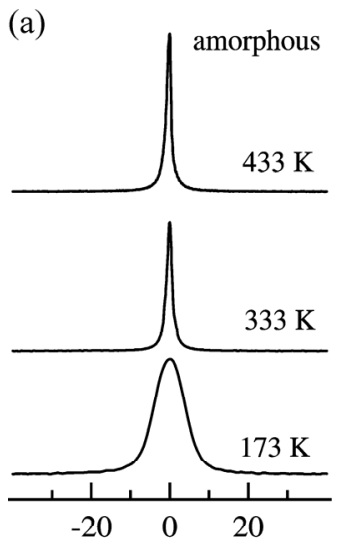

frequency $(\mathrm{kHz})$ (b)
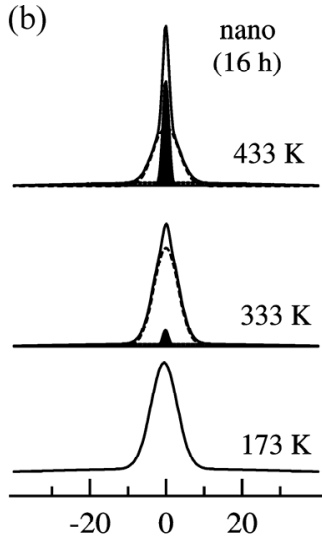

frequency $(\mathrm{kHz})$

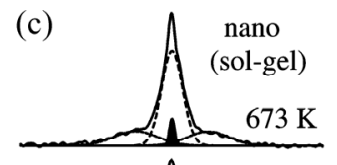

(d) micro
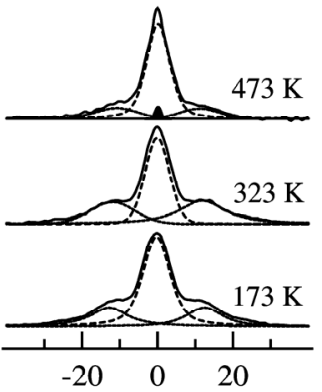

frequency $(\mathrm{kHz})$
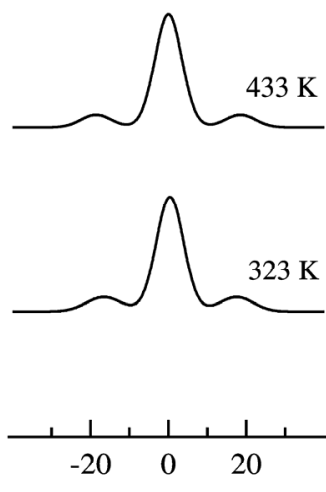

frequency $(\mathrm{kHz})$

Fig. 7 Temperature dependent ${ }^{7} \mathrm{Li}$ NMR spectra $\left(\omega_{0} / 2 \pi=77.7 \mathrm{MHz}\right)$ of (a) amorphous, (b) mechanically prepared nanocrystalline, (c) chemically (sol-gel) prepared nanocrystalline, and (d) microcrystalline $\mathrm{LiNbO}_{3}$ delivered by Alfa Aesar. Narrow contributions $A_{\mathrm{f}}$ of the respective nanocrystalline samples are shown by filled areas, whereas the broad contributions are indicated by dashed lines. Quadrupole contributions to the NMR line of the sol-gel nanocrystalline material are represented by solid lines.

the Li spectra are composed of a central NMR line and two satellite contributions with minor intensity shifted to lower and higher resonance frequencies. The splitting of the central line into three NMR lines is simply due to the interaction of the quadrupole moment of the nuclei with a nonvanishing electric field gradient produced by the electric charge distributions in the neighbourhood of the Li site.

In the case of amorphous $\mathrm{LiNbO}_{3}$ (Fig. 7 (a)) mainly the central transition is observed when the NMR signal is recorded via the solid-echo pulse sequence. With increasing temperature the central line starts to narrow due to the onset of Li motions with jump rates in the $\mathrm{kHz}$ range. Its shape can be described with a Gaussian line at low temperatures, i. e., in the rigid lattice regime below $200 \mathrm{~K}$, and with a Lorentzian line at higher temperatures above, e. g., $300 \mathrm{~K}$.

The same description is valid also for the central transition of the microcrystalline sample (Fig. 7 (d)). The onset of motional narrowing for microcrystalline $\mathrm{LiNbO}_{3}$ starts at about $650 \mathrm{~K}$, thus being shifted by some hundreds of degrees to higher temperatures due to the much lower Li diffusivity as compared to that in the highly disordered sample (see below and $c f$. Fig. 9). In the powder spectra of coarse grained $\mathrm{LiNbO}_{3}$ a pair of well-defined quadrupole satellites appears next to the central NMR transition. This quadrupole splitting was also observed for single-crystalline $\mathrm{LiNbO}_{3}$.The observation of satellite transitions is related to the well-defined crystalline, less- 
defective structure of coarse grained $\mathrm{LiNbO}_{3}$ as compared to that of amorphous $\mathrm{LiNbO}_{3}$. In the latter one, because of the disordered structure, the satellite lines are broadened or smeared out due to a relatively large distribution of electric field gradients and thus quadrupole coupling constants. In amorphous $\mathrm{LiNbO}_{3}$ the quadrupole contributions can hardly be seen, forming only a broad background.

The same holds true for the nanocrystalline sample which was prepared by ball-milling and which consists of a large amount of amorphous regions (Fig. 7 (b)). Contrary to that, the sol-gel prepared nanocrystalline sample shows a higher degree of crystallinity so that the satellite transitions can be clearly seen (Fig. 7 (c)).

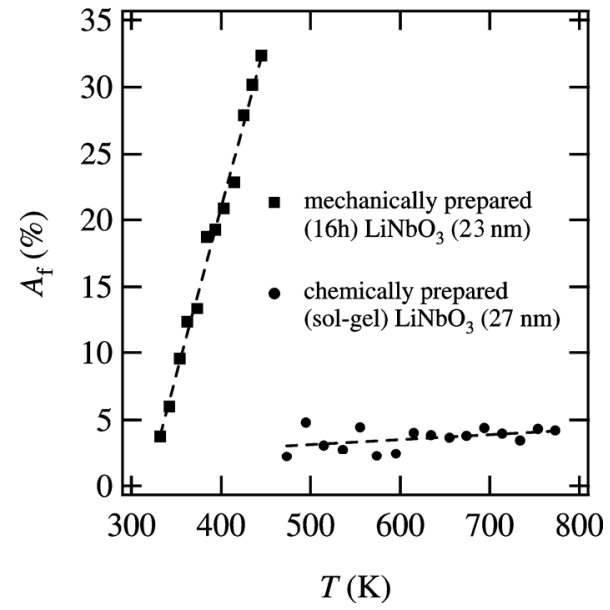

Fig. 8 Area fraction $A_{\mathrm{f}}(\%)$ of the narrow ${ }^{7} \mathrm{Li}$ NMR contribution of the total area of the NMR line as a function of temperature for the two nanocrystalline materials prepared either mechanically by ball-milling for $16 \mathrm{~h}(\boldsymbol{\square})$ or chemically via the sol-gel technique $(\bullet)$.

Thus, the degree of disorder can be probed by studying satellite contributions to the respective spectra. The two nanocrystalline samples take a medial position between amorphous and microcrystalline $\mathrm{LiNbO}_{3}$; whereas the chemically prepared one is structurally more related to the microcrystalline sample, the ball-milled material is similar to the amorphous one.

In addition to structural characteristics also dynamical aspects can excellently be studied via recording ${ }^{7} \mathrm{Li}$ NMR spectra. ${ }^{6,21,22,27}$ Contrary to the amorphous and microcrystalline material, the central line of the $16 \mathrm{~h}$ high-energy ball-milled sample shows two contributions at the same fre- 
quency when a temperature of about $330 \mathrm{~K}$ is attained. The narrow contribution, which can be described with a Lorentzian shape, is attributed to the fast subset of Li ions located in the structurally disordered interfacial regions. The broad Gaussian contribution represents the Li ions, which are still immobile at this temperature. ${ }^{6}$ Similar results were obtained for the nanocrystalline anionic conductor $\mathrm{CaF}_{2} \cdot{ }^{22,42}$ The NMR results confirm, that this nanocrystalline $\mathrm{LiNbO}_{3}$ sample is characterized by a heterogeneous, i.e., two-component structure of crystalline grains and amorphous grain boundaries.

The fraction of mobile ions is given by the area of the narrow contribution relative to the area of the total resonance line. Fig. 8 shows the relative contribution $A_{\mathrm{f}}$ of the narrow NMR line as a function of temperature. At about $450 \mathrm{~K}$ the fraction of mobile ions, dominating the Li transport process in this material, reaches a value of nearly $35 \%$ being fairly consistent with the results from EXAFS measurements (see above and ref. 38). To avoid any grain growth the NMR experiments had to be restricted to an upper temperature limit of approximately $450 \mathrm{~K}$ (see above, sec. 2.2).

In contrast to this result, the heterogeneous motional narrowing of the sol-gel prepared sample is much less pronounced than it is in the case of the milled sample. A narrow contribution cannot be separated before a temperature of about $470 \mathrm{~K}$ is reached. This narrow NMR line shows a constant relative contribution of about $4 \%$ indicating once more the relatively thin grain boundary regions of sol-gel prepared nanocrystalline $\mathrm{LiNbO}_{3}$.

In order to ascertain the Li dynamics in a more quantitative manner, the linewidths of the different forms of $\mathrm{LiNbO}_{3}$ were determined as a function of temperature. Fig. 9 shows this temperature dependence. For amorphous $\mathrm{LiNbO}_{3}$ the NMR line starts to narrow at about $225 \mathrm{~K}$, whereas the onset of motional narrowing of the microcrystalline material is shifted to about $700 \mathrm{~K}$. The onset temperature $T_{\text {onset }}$ is related via the empirical expression of Waugh and Fedin ${ }^{43}$

$$
E_{\mathrm{A}}^{\mathrm{MN}} / \mathrm{eV}=1.617 \times 10^{-3} \cdot T_{\text {onset }} / K
$$

with the activation energy $E_{\mathrm{A}}^{\mathrm{MN}}$ of the Li hopping process. The activation energy is estimated to be about $0.32 \mathrm{eV}$ for amorphous $\mathrm{LiNbO}_{3}$ and about $0.46 \mathrm{eV}$ for the ball-milled sample, whereas it is much higher for the microcrystalline source material, $1.07 \mathrm{eV} .^{6,27,44}$ Motional narrowing of the ball- 
(a)

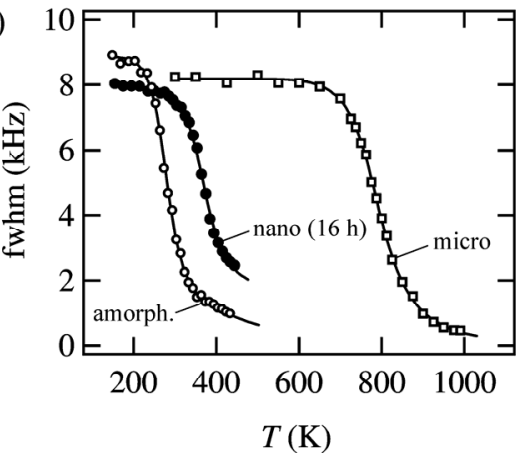

(b)

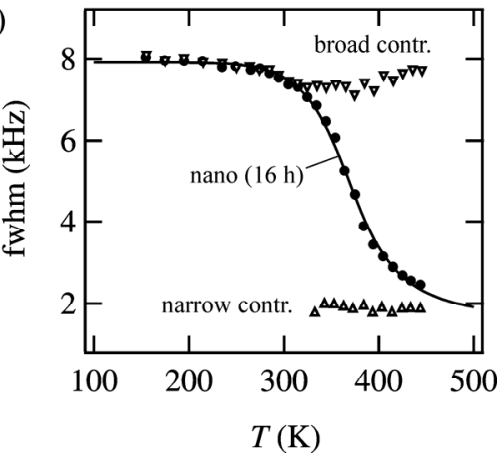

Fig. 9 (a) ${ }^{7}$ Li NMR linewidths (fwhm: full width at half maximum of the overall NMR line) as a function of temperature for amorphous $(\circ)$ and microcrystalline $(\square) \mathrm{LiNbO}_{3}$, as well as for the nanocrystalline materials prepared by highenergy ball-milling of the microcrystalline material for $16 \mathrm{~h}(\bullet)$. The linewidths were obtained from ${ }^{7} \mathrm{Li}$ spectra which were acquired at a resonance frequency of $77.7 \mathrm{MHz}$ and using the solid-echo pulse sequence. (b) Decomposition of the ${ }^{7} \mathrm{Li}$ NMR line nanocrystalline $\mathrm{LiNbO}_{3}$ prepared by ball-milling for $16 \mathrm{~h}$ into a broad $(\nabla)$ and narrow contribution $(\triangle)$. The linewidth of the entire NMR line $(\bullet)$ of Fig. 9 (a) is shown for comparison. In both figures solid lines are to guide the eye. The data of microcrystalline $\mathrm{LiNbO}_{3}$ are taken from ref. 6.

milled material is more comparable to that of amorphous $\mathrm{LiNbO}_{3}$, as quite recently has also been reported by Chadwick et al. ${ }^{28}$ In the range below $470 \mathrm{~K}$ the sol-gel prepared nanocrystalline material reveals no sharp decrease in linewidth with increasing temperature, like the other samples do, since its Li diffusivity is not dominated by fast diffusion pathways located in the interfacial regions. Thus, the same trend of Li diffusivity is found by NMR solid-echo experiments as was obtained by conductivity measurements (see sec. 4.1 and ref. 23). In order to analyze the twocomponent shape of the ${ }^{7} \mathrm{Li}$ NMR spectra of the ball-milled nanocrystalline sample, the linewidths of the two distinct contributions are shown separately in Fig. 9 (b) as a function of temperature. The emergence of the narrow contribution and the narrowing of the total line occurs simultaneously at about $330 \mathrm{~K}$. At this temperature the NMR linewidth of the corresponding amorphous sample is already extremely narrowed.

Irrespective of grain growth we have intentionally also performed some high-temperature measurements up to $750 \mathrm{~K}$ on the sol-gel prepared nanocrystalline sample (not shown here, see ref. 37 for details). The narrow contribution started to be separable only above $470 \mathrm{~K}$. It has a fwhm comparable to that of the amorphous form. As expected, it showed only a minor effect on the narrowing of the total line due to its nearly temperature independent small area fraction $A_{\mathrm{f}}$ (Fig. 8).

\subsection{2 ${ }^{7} \mathrm{Li}$ NMR spin-lattice relaxation}


In addition to the analysis of solid-echo NMR spectra, the effect of grain size and microstructure on the Li diffusion process was also investigated by ${ }^{7} \mathrm{Li}$ NMR spin-lattice relaxation measurements. In Fig. 10 the ${ }^{7} \mathrm{Li}$ NMR spin-lattice relaxation rates $T_{1}^{-1}\left(T^{-1}\right)$ of the amorphous, the two nanocrystalline and the microcrystalline samples are shown. The data were recorded at a resonance frequency of 77.7 MHz. Except for the nanocrystalline sample prepared by the sol-gel route, the plots of $T_{1}^{-1}$ $v s$. reciprocal temperature consist of a superposition of a diffusion induced and a background contribution $^{27}$ :

$$
T_{1}^{-1}=T_{1 \mathrm{diff}}^{-1}+T_{1 \mathrm{bgr}}^{-1}
$$

Whereas the diffusion induced spin-lattice relaxation rates $T_{1 \text { diff }}^{-1}$ follow Arrhenius behaviour,

$$
T_{1 \text { diff }}^{-1}\left(T^{-1}\right)=A^{\prime \prime} \exp \left(E_{\mathrm{A}, \mathrm{SLR}} / k_{\mathrm{B}} T\right)
$$

which represents the low-temperature flank of the characteristic $T_{1 \text { diff }}^{-1}\left(T^{-1}\right)$-peak ${ }^{22}$, the background

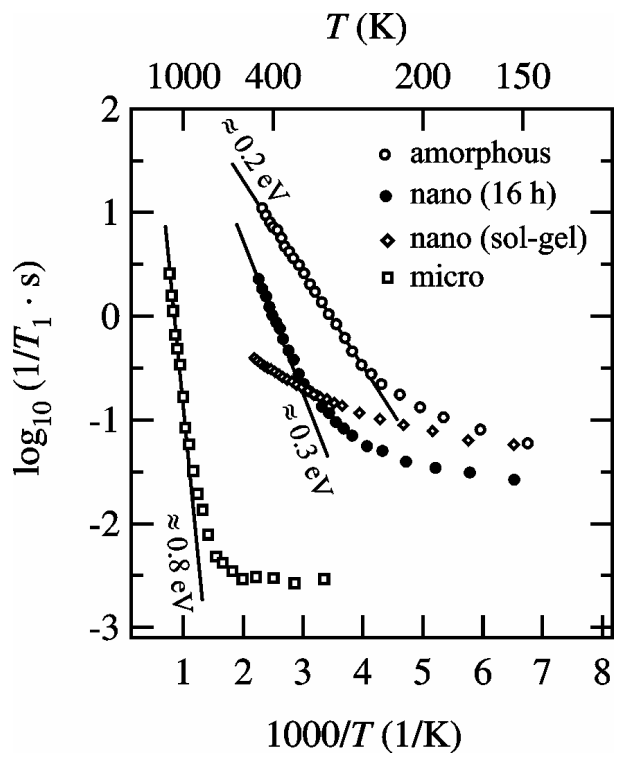

Fig. $10{ }^{7} \mathrm{Li}$ NMR spin-lattice relaxation rates vs. reciprocal temperature for amorphous $(\circ)$ and nanocrystalline $\mathrm{LiNbO}_{3}$ which was either prepared by ballmilling for $16 \mathrm{~h}(\bullet)$ or by sol-gel synthesis $(\diamond)$. The data of commercially available microcrystalline $\mathrm{LiNbO}_{3}(\square)$ are shown for comparison. The data of microcrystalline $\mathrm{LiNbO}_{3}$ are taken from ref. 6 . 
contribution $T_{1 \text { bgr }}^{-1}$ shows a weaker-than-activated temperature behaviour which can usually be described with a simple power law $T_{1 \mathrm{bgr}}^{-1}\left(T^{-1}\right) \propto T^{\kappa}$, see ref. 27 . The background relaxation is mostly caused by lattice vibrations and/or paramagnetic impurities. However, in the case of microcrystalline $\mathrm{LiNbO}_{3}$ this background contribution, which shows up below $T=500 \mathrm{~K}$ seems to be temperature independent. Above $800 \mathrm{~K}$ the relaxation rate is predominantly induced by diffusion of Li ions with an activation energy of about $0.8 \mathrm{eV}$. The diffusion induced relaxation rate is proportional to the mean jump rate $\tau^{-1}$ of the Li ions. The corresponding diffusion induced $T_{1 \text { diff }}^{-1}\left(T^{-1}\right)$ flank of the amorphous material is shifted to much lower temperatures, verifying much faster Li diffusion in this material. Above room temperature the relaxation rates are mainly induced by Li diffusion, the activation energy is drastically reduced and turned out to be about $0.22 \mathrm{eV}$. Recently, we reported also on ${ }^{7} \mathrm{Li}$ NMR relaxation measurements on the amorphous sample which was prepared by partial hydrolysis of the lithium niobium double alkoxide ( $c f$. sec. 2.1 and ref. 27). For that material an activation energy of about $0.27 \mathrm{eV}$ was found by means of ${ }^{7} \mathrm{Li}$ NMR relaxation. ${ }^{27}$ Such small differences in activation energies are expected since both materials are prepared by slightly different preparation routes ( $c f$. sec. 2.1), and will not further be discussed in the present paper.

Similar to the results of amorphous $\mathrm{LiNbO}_{3}$, also the diffusion induced flank of the heterogeneously structured, ball-milled nanocrystalline $\mathrm{LiNbO}_{3}$ sample is shifted to lower temperatures in comparison with that of the coarse grained (microcrystalline) material. The activation energy of about $0.3 \mathrm{eV}$ for the nanocrystalline material is comparable to that obtained for the completely disordered sample.

As expected, the spin-lattice relaxation behaviour of the chemically prepared nanocrystalline sample is different from that of the mechanically prepared one. In the investigated temperature range up to $T=460 \mathrm{~K}$ no sharp increase in $T_{1}^{-1}\left(T^{-1}\right)$ is observed. Instead of that, only a nondiffusive relaxation background is detected whose temperature dependence is similar to that which is observed for the nanocrystalline sample prepared by $16 \mathrm{~h}$ ball-milling. Thus, ${ }^{7} \mathrm{Li}$ NMR spinlattice relaxation measurements confirm once more that the overall Li diffusivity in the sol-gel prepared nanocrystalline material is much lower than that in the homogeneously or heterogeneously 
disordered samples, i.e. the amorphous and the nanocrystalline sample prepared by high-energy ball-milling, respectively.

\section{Conclusion}

$\mathrm{Li}$ transport in two differently prepared nanocrystalline $\mathrm{LiNbO}_{3}$ samples with nearly the same average particle size in the range between 20 and $30 \mathrm{~nm}$ was extensively investigated by means of impedance spectroscopy and solid-state ${ }^{7} \mathrm{Li}$ NMR spectroscopy. The results were compared with those obtained for the micro- and single-crystalline as well as the amorphous form of $\mathrm{LiNbO}_{3}$. Nanocrystalline $\mathrm{LiNbO}_{3}$ was either prepared mechanically by high-energy ball-milling or chemically via the sol-gel technique. In both cases, nanostructuring results in enhanced Li diffusivity as compared to the cation transport in coarse grained or single-crystalline lithium niobate.

It was unambiguously shown by HRTEM images and EXAFS measurements that the grain boundaries of nanocrystalline $\mathrm{LiNbO}_{3}$ prepared by ball-milling have amorphous structure. These results are fully corroborated by the data obtained from impedance spectroscopy and NMR relaxation experiments on the four different forms of $\mathrm{LiNbO}_{3}$. $\mathrm{Li}$ ions which reside in the disordered interfacial regions have access to fast diffusion pathways with low activation energies. As could be shown by dc-conductivity measurements and ${ }^{7} \mathrm{Li} \mathrm{NMR}$, the overall Li transport is dominated by these fast charge carriers. However, although Li diffusivity in sol-gel prepared nanocrystalline is also enhanced, its Li transport behaviour is more related to that of the coarse grained material than to amorphous $\mathrm{LiNbO}_{3}$. HRTEM images as well as XRD results show, that the grain-boundaries are thinner and have a much more ordered structure than in the ball-milled samples. Thus, we could clearly show, that transport properties are strongly related to the nature of the preparationdependent grain boundary microstructure.

The results concerning the microstructure of interfacial regions in $\mathrm{LiNbO}_{3}$ are in agreement with recently drawn conclusions from EXAFS measurements on other, but Li free, nanocrystalline oxides like sol-gel prepared $\mathrm{SnO}_{2}$, refs. 45 and 46, sol-gel and mechanically prepared $\mathrm{ZrO}_{2}$, ref. 47, as well as Y-stabilised cubic $\mathrm{ZrO}_{2}$, ref. 48, prepared by polymer spin coating.

The sol-gel technique, like other soft-chemistry routes, gives access to nanocrystalline materials with grain boundaries similar to those in bulk materials, whereas ball-milling leads to oxides hav- 
ing a larger level of disorder in the interfacial regions. In cases where larger amounts of a highly conducting material are needed, the latter preparation route ${ }^{30}$ may be advantageous. It also allows easy extension to composite nanocrystalline ceramics. ${ }^{5,7,9}$

\section{Acknowledgement}

The authors wish to thank C. Rüscher, H. Behrens and T. Gesing (Institute of Mineralogy, Hannover University) as well as M. Klüppel, J. Meier and B. Huneke (German Institute for Rubber Technology, Hannover) for their help and valuable technical support. The cooperation of D. Bork, R. Winter, D.-M. Fischer, N. Greaves and A. Dent in the EXAFS project is highly appreciated. M.M. would like to acknowledge a grant of the Center of Solid State Chemistry and New Materials (Hannover University). P.H. is grateful to G.E. Murch and I.V. Belova for their hospitality at the University of Newcastle (Callaghan, Australia), where part of this article was written.

\section{References}

1 S. Chandra, Superionic Solids, Principles and Applications, North-Holland, Amsterdam, 1981.

2 Lithium Ion Batteries, edited by M. Wakihara and O. Yamamoto, Wiley-VCH, Weinheim, 1998.

3 M. S. Whittingham, Chem. Rev., 2004, 104, 4271.

4 J.-M. Tarascon and M. Armand, Nature, 2001, 414, 359.

$5 \quad$ P. Heitjans and S. Indris, J. Phys.: Condens. Matter, 2003, 15, R1257.

6 D. Bork and P. Heitjans, J. Phys. Chem. B, 1998, 102, 7303; D. Bork and P. Heitjans, J. Phys. Chem. B, 2001, 105, 9162 .

7 S. Indris, P. Heitjans, H. E. Roman and A. Bunde, Phys. Rev. Lett., 2000, 84, 2889.

8 S. Indris and P. Heitjans, J. Non-Cryst. Solids, 2000, 307, 555.

9 M. Wilkening, S. Indris and P. Heitjans, Phys. Chem. Chem. Phys., 2003, 5, 2225.

10 W. Puin and P. Heitjans, Nanostruct. Mater. 1995, 6, 885; W. Puin, S. Rodewald, R. Ramlau, P. Heitjans and J. Maier, Solid State Ion. 2000, 131, 159.

11 U. Brossmann, R. Würschum, U. Södervall and H.-E. Schaefer, Nanostruct. Mater., 1999, $12,871$.

12 P. Knauth, J. Solid State Electrochem., 2002, 147115.

13 P. Knauth and H. L. Tuller, Solid State Ion., 2000, 136-137, 1215.

14 Y.-M. Chiang, E. B. Lavik, I. Kosacki, H.L. Tuller and J.Y. Ying, J. Electroceram., 1997, 1, 7.

15 A. Tschöpe, E. Sommer R. and Birringer, Solid State Ion., 2001, 139, 255. 
17 C.-W. Nan, A. Tschöpe, S. Holten, H. Kliem and R. Birringer, J. Appl. Phys., 1999, 85, 7735.

18 G. Li, L. Li, S. Feng, M. Wang, L. Zhang and X. Yao, Adv. Mater., 1999, 11, 146.

19 P. Mondal, A. Klein, W. Jaegermann and H. Hahn, Solid State Ion., 1999, 118, 331.

20 U. Brossmann, G. Knöner, H.-E. Schaefer and R. Würschum, Rev. Adv. Mater. Sci., 2004, 6, 7.

21 P. Heitjans, S. Indris and M. Wilkening, Solid-State Diffusion and NMR in: Diffusion Fundamentals edited by J. Kärger, F. Grinberg and P. Heitjans, Leipziger Universitätsverlag, Leipzig, 2005, pp. 226-245.

22 P. Heitjans, A. Schirmer, S. Indris, NMR and $\beta$-NMR Studies of Diffusion in Interface-Dominated and Disordered Solids in: Diffusion in Condensed Matter - Methods, Materials, Models, edited by P. Heitjans and J. Kärger, Springer, Berlin/Heidelberg, 2005, pp. 367-415.

23 M. Masoud and P. Heitjans, Defect Diffus. Forum, 2005, 237-240, 1016.

24 S. Lanfredi and A. Rodrigues, J. Appl. Phys., 1999, 86, 2215.

25 A. Glass, K. Nassau and T. Negran, J. Appl. Phys., 1978, 49, 4808.

26 K. Nassau, J. Non-Cryst. Solids, 1980, 42, 423.

27 M. Wilkening, D. Bork, S. Indris and P. Heitjans, Phys. Chem. Chem. Phys., 2002, 4, 3246.

28 A. V. Chadwick, M. J. Pooley and S. L. P. Savin, Phys. Stat. Sol., 2005, 2, 302.

29 S. Ono, H. Mochizuki and S. Hirano, J. Cer. Soc. Jap., 1996, 104, 574.

30 S. Indris, D. Bork and P. Heitjans, J. Mater. Synth. Proc., 2000, 8, 245.

31 H. Gleiter, Prog. Mater. Sci., 1989, 33, 223.

32 V. V. Srdic, M. Winterer, G. Miehe and H. Hahn, Nanostruct. Mater., 1999, 12, 95.

33 H. Natter, M. Schmelzer, S. Janßen and R. Hempelmann, Ber. Bunsenges. Phys. Chem., 1997, $101,1706$.

34 Nanophase Materials, editec by G. Hadjypanayis and R. W. Siegel, Kluwer Academic Publishers, Netherlands, 1994.

35 H. P. Klug and L. E. Alexander, X-ray diffraction procedures, Wiley, New York, 1974; P. Scherrer, Göttinger Nachrichten, 1918, 2, 98.

36 W. Rachinger, J. Sci. Instr., 1948, 25, 254; B. Warren, X-ray diffraction, Addison Wesley, New York, 1969.

37 M. Masoud, PhD thesis, Hannover University, 2005.

38 M. Masoud, P. Heitjans, D. Bork, R. Winter, N. Greaves, D.-M. Fischer, A. Dent, submitted.

39 M. J. Pooley and A. V. Chadwick, Rad. Effect. Defects, 2003, 158, 197.

40 E. Fukushima, S. B. W. Roeder, Experimental Pulse NMR, Addison-Wesley, Reading, 1981.

41 M. Niederberger, N. Pinna, J. Polleux and M. Antonietti, Angew. Chemie Int. Ed., 2004, 43, 2270 
42 W. Puin, P. Heitjans, W. Dickenscheid and H. Gleiter, Defects in Insulating Materials edited by O. Kanert and J. Spaeth, Singapore: World Scientific, 1993.

43 J. S. Waugh and E. I. Fedin, Sov. Phys. Solid State, 1963, 4, 1633.

44 Y. Xia, N. Machida, X. Wu, Ch. Lakeman, L. van Wüllen, F. Lange, C. Levi and H. Eckert, J. Phys. Chem. B, 1997, 101, 9180.

45 S. Davis, A. Chadwick and J. Wright, J. Phys. Chem. B, 1997, 101, 9901;

46 A. V. Chadwick, Rad. Effect. Defects, 2003, 158, 21.

47 A. V. Chadwick, M. J. Pooley, K. E. Rammutla, S. L. P. Savin and A. Rougier, J. Phys.: Condens. Matter, 2003, 15, 431 .

48 G. Rush, A. V. Chadwick, I. Kosacki and U. Anderson, J. Phys. Chem. B, 2000, 104, 9597. 